\title{
A novel fully human antitumour immunoRNase targeting ErbB2-positive tumours
}

\author{
M Borriello', P Laccetti', G Terrazzano ${ }^{2,3}$, G D'Alessio' and C De Lorenzo*,I \\ 'Dipartimento di Biologia Strutturale e Funzionale, Università Federico II, via Cinthia, Napoli 80 I 26, Italy; ${ }^{2}$ Dipartimento di Patologia e Biologia Cellulare \\ e Molecolare, Università Federico II, via Pansini, Napoli 80 I3I, Italy; ${ }^{3}$ Dipartimento di Chimica, Università della Basilicata, Via N. Sauro, 85, Potenza \\ 85100 , Italy
}

BACKGROUND: ErbB2 is an attractive target for immunotherapy, as it is a tyrosine kinase receptor overexpressed on tumour cells of different origin, with a key role in the development of malignancy. Trastuzumab, the only humanised anti-ErbB2 antibody currently used in breast cancer with success, can engender cardiotoxicity and a high fraction of patients is resistant to Trastuzumab treatment. METHODS: A novel human immunoRNase, called anti-ErbB2 human compact antibody-RNase (Erb-hcAb-RNase), made up of the compact anti-ErbB2 antibody Erbicin-human-compact Antibody (Erb-hcAb) and human pancreatic RNase (HP-RNase), has been designed, expressed in mammalian cell cultures and purified. The immunoRNase was then characterised as an enzymatic protein, and tested for its biological actions in vitro and in vivo on ErbB2-positive tumour cells.

RESULTS: Erb-hcAb-RNase retains the enzymatic activity of HP-RNase and specifically binds to ErbB2-positive cells with an affinity comparable with that of the parental Erb-hcAb. Moreover, this novel immunoRNase is endowed with an effective and selective antiproliferative action for ErbB2-positive tumour cells both in vitro and in vivo. Its antitumour activity is more potent than that of the parental Erb-hcAb as the novel immunoconjugate has acquired RNase-based cytotoxicity in addition to the inhibitory growth effects, antibody-dependent and complement-dependent cytotoxicity of Erb-hcAb.

CONCLUSION: Erb-hcAb-RNase could be a promising candidate for the immunotherapy of ErbB2-positive tumours.

British Journal of Cancer (201 I) I 04, I7I6-1723. doi:I0.1038/bjc.20 I I.I46 www.bjcancer.com

Published online 10 May 2011

(C) 20II Cancer Research UK

Keywords: immunotherapy; ErbB2/HER2; immunoRNase; breast cancer; Trastuzumab

Immunotherapy is a precious strategy to overcome the limits of the conventional anticancer treatments. Indeed, targeting cancer cells via antibodies specific for tumour-associated surface proteins could fulfil the lack of selectivity of radiotherapy and chemotherapy, and is a new interesting biomedical approach as it combines the rational drug design with the progress in understanding cancer biology.

To date, several humanised monoclonal antibodies (MAbs) have achieved FDA approval, and an increasing number is undergoing clinical evaluation (Harris, 2004; Adams and Weiner, 2005; Venkiteshwaran, 2009; Li and Zhu, 2010). A successful example of an approved humanised antibody is represented by Trastuzumab, the only humanised antibody widely used against ErbB2-positive carcinomas for immunotherapy (Stebbing et al, 2000).

ErbB2 is an attractive target for immunotherapy, as it is a transmembrane tyrosine kinase receptor, overexpressed on tumour cells of different origin, with a key role in the development of malignancy (Slamon et al, 1987). Trastuzumab is currently used with success for breast cancer therapy; however, it can engender cardiotoxicity and a high fraction of breast cancer patients is resistant to Trastuzumab treatment (Seidman et al, 2002; Nahta et al, 2006).

*Correspondence: Dr C De Lorenzo; Email: cladelor@unina.it Received 18 March 20II; revised 5 April 2011; accepted 8 April 20II; published online I0 May 20I I
Furthermore, also carcinomas with a high expression of ErbB2, such as non-small cell lung carcinoma, gastric and prostatic tumours, have been found to be resistant or much less sensitive to Trastuzumab treatment (Agus et al, 1999; Gong et al, 2004). When Trastuzumab was used in combination with chemotherapy, some benefits have been shown in clinical trials for patients, such as those with ErbB2-positive advanced gastric cancer (Jørgensen, 2010), but cardiac dysfunction has also been observed more frequently (Seidman et al, 2002).

A significant addition to the anticancer arsenal has been the construction of a new anti-ErbB2 immunoagent derived from a human, per se cytotoxic single-chain antibody fragment (scFv) named Erbicin (De Lorenzo et al, 2002b), and a human Fc domain from a human IgG1. This led to a fully human antitumour antibody, designed to be a reduced version of an IgG, with the antiproliferative effect of the scFv moiety on tumour target cells, combined with the ability of the Fc moiety to induce both antibody-dependent cellular cytotoxicity (ADCC) and complement-dependent cytotoxicity (CDC).

The engineered antibody has been called Erbicin-humancompact Antibody (Erb-hcAb) for its 'compact' size ( $100 \mathrm{kDa})$, compared with the full size $(155 \mathrm{kDa})$ of a natural IgG. The smaller size should promote an increased extravascular diffusion and tumour penetration.

It has been reported that Erb-hcAb is capable of selective binding to malignant ErbB2-positive cells and of inhibiting their 
growth in vitro and in vivo, with no effects on ErbB2-negative cells. Moreover, Erb-hcAb is endowed with both ADCC and CDC cytotoxic effects, whereas Trastuzumab lacks the ability of inducing CDC (De Lorenzo et al, 2004b).

More recently, it has been shown that Erb-hcAb does not display the cardiotoxic effects of Trastuzumab in vitro on rat cardiomyocytes and in vivo on a mouse model (Riccio et al, 2009), whereas Trastuzumab was found to be strongly toxic. This difference was found to be due to the different mechanism of action of the two antibodies: Trastuzumab, at difference with Erb-hcAb, induces apoptosis in cardiac cells (Riccio et al, 2009). Finally, Erb-hcAb is active in vitro and in vivo against some Trastuzumab-resistant, ErbB2-positive breast cancer cell lines (Gelardi et al, 2010).

Targeted therapy can be accomplished also by using MAbs equipped with radionuclides or toxins (Pastan and FitzGerald, 1991; Carter, 2001). Immunotoxins (ITs) are anticancer agents made up of a recombinant antibody or an antibody fragment directed towards a unique cell surface protein and a potent bacterial toxin capable of inducing the death of target cells (Reiter and Pastan, 1998; Zhang et al, 2007).

However, problems have been encountered in clinical trials, especially for the toxicity and immunogenicity of the bacterial or plant toxins used for the ITs (Weiner et al, 1989; Schindler et al, 2001).

As an alternative to ITs, ImmunoRNases (IRs) have been proposed as more immunocompatible immunoagents. These are fusion proteins in which the toxin has been replaced by a ribonuclease. Mammalian RNases are expected to be not immunogenic and not systemically toxic, as they are pro-toxins, which become toxic only upon their internalisation in target cells mediated by the antibody moiety (Rybak and Newton, 1999; De Lorenzo et al, 2002a; De Lorenzo and D'Alessio, 2008).

A fully human immunoRNase, Erbicin-human-RNase (ErbhRNase), was constructed through the fusion of Erbicin with human pancreatic RNase (HP-RNase or RNase 1) (De Lorenzo et al, 2004a). The chimeric protein was found to retain the highbinding affinity to ErbB2-positive cells of Erbicin and the enzymatic activity of native HP-RNase. When tested in vitro on a series of malignant cells, Erb-hRNase was found to discriminate between target and non-target cells, and to specifically inhibit the proliferation of ErbB2-positive cells, with a stronger cytotoxicity on cells with a higher level of ErbB2. Its antitumour activity has been also demonstrated in vivo on mice implanted with ErbB2positive tumours (De Lorenzo et al, 2004a; De Lorenzo and D’Alessio, 2009).

The antitumour action of Erb-hRNase is dependent on the ability of this molecule to reach the cytosol and degrade RNA, but it is somewhat limited by the finding (De Lorenzo et al, 2007) that the enzymatic activity of Erb-hRNase is inhibited by the RNase inhibitor (RI).

In order to obtain a novel product, which is expected to be superior to the immunoagents currently used in the therapy of breast cancer, a novel immunoRNase has been designed, in which an Erbicin-based scFv-Fc (Erb-hcAb, see above) replaces the scFv of Erb-hRNase. The latter should have the following advantages: the ability of inducing both ADCC and CDC, in addition to the RNase-based cytotoxicity; a prolonged half-life, due to its higher molecular size and the presence of the $\mathrm{Fc}$ and an increased avidity due to the presence of two scFv moieties. Furthermore, this novel immunoagent could be resistant to the action of RI, due to the steric hindrance generated by the presence of the larger antibody moiety.

Here, we report on the construction and characterisation of such novel fully human immunoRNase, made up of Erb-hcAb and HP-RNase, expressed in mammalian cell cultures.

This new immunoconjugate, called anti-ErbB2 human compact antibody-RNase (Erb-hcAb-RNase), has shown to fully retain the binding ability, ADCC and CDC properties of Erb-hcAb and to acquire the RNase activity of its enzymatic moiety, thus inhibiting tumour cell proliferation in vitro and in vivo more efficiently than the parental Erb-hcAb.

\section{MATERIALS AND METHODS}

\section{Cell cultures and antibodies}

The SKBR3 cell line from human breast cancer and the A431 cell line from human epidermoid carcinoma were cultured in RPMI 1640 (Gibco BRL, Life Technologies, Paisley, UK). The TUBO cell line from a BALB-neu $\mathrm{T}$ mouse-derived mammary lobular carcinoma (kindly provided by Dr G Forni, University of Turin, Italy) was grown in DMEM (Gibco BRL). The media were supplemented with $10 \%$ fetal bovine serum (20\% for TUBO cells), $50 \mathrm{U} \mathrm{ml}^{-1}$ penicillin and $50 \mu \mathrm{g} \mathrm{ml}^{-1}$ streptomycin (all from Gibco BRL).

The antibodies used were Trastuzumab (Genentech, South San Francisco, CA, USA); affinity-isolated IgGs from a rabbit antiHP-RNase antiserum (from Igtech, Salerno, Italy); horseradish peroxidase (HRP)-conjugated goat anti-rabbit immunoglobulins (Pierce, Rockford, IL, USA); HRP-conjugated goat anti-human affinity-isolated IgGs (Fc specific) (Sigma, St Louis, MO, USA); Erb-hcAb was produced by PER.C6 cells (Crucell NV, Leiden, Netherlands) transfected with the recombinant vector (De Lorenzo et al, 2004b), and purified as previously described (De Lorenzo et al, 2004b).

\section{Peripheral blood lymphocytes}

Peripheral blood lymphocytes (PBL) were obtained from peripheral blood mononuclear cells isolated by centrifugation on Lymphoprep gradients (Axis Shield PoC AS, Oslo, Norway) from normal donor buffy coats obtained from the Blood Bank of the Medical School of the University of Naples 'Federico II'. After the separation, PBL were washed twice and incubated in RPMI 1640 medium (Gibco BRL) for $2 \mathrm{~h}$ at $37^{\circ} \mathrm{C}$ to remove adherent cells. The non-adherent cells were used as natural cytotoxic effectors without any additional treatment.

\section{Construction and production of the anti-ErbB2 Erb-hcAb- RNase}

The cDNA coding for the human compact antibody Erb-hcAb (De Lorenzo et al, 2004b) was amplified from plasmid pIg1plus (R \& D Systems, Minneapolis, MN, USA) by PCR using as forward and reverse primers oligonucleotides containing at their $5^{\prime}$ and $3^{\prime}$ ends an SalI and an NotI site, respectively: 5'-ACGCGTCGACCAGGTGC AGCTGTTG- ${ }^{\prime}$; 5' $^{\prime}$-ATAAGAATGCGGCCGCTTTACCCGGAGACA GG-3'. The PCR fragment was then digested with SalI and NotI (New England Biolabs, Hertfordshire, UK) for cloning into the eukaryotic pCMV-endoplasmic reticulum (ER)-myc expression vector (Invitrogen, Carlsbad, CA, USA) downstream to the leader ER signal peptide sequence.

In order to obtain the chimeric construct, the gene fragment coding for the HP-RNase was cloned downstream to the Erb-hcAb sequence by inserting a spacer encoding an 11-amino-acid peptide linker (AAASGGPEGGS) between the $\mathrm{scFv}-\mathrm{Fc}$ and the RNase. The cDNA coding for HP-RNase and the peptide spacer, previously generated (De Lorenzo et al, 2004a) with NotI restriction sites at its $3^{\prime}$ and $5^{\prime}$ ends, was digested with NotI and cloned into the corresponding site of pCMV-ER-myc vector downstream to the sequence encoding the human Erb-hcAb.

The correct directional insertion of the RNase gene in the NotI sites was assessed by digestion with suitable endonucleases (New England Biolabs). Sequence analyses confirmed the expected DNA sequence.

The fusion protein was produced by transfecting $293 \mathrm{~T}$ (human embryonic kidney) cells with the recombinant vector. In brief, cells 
grown in DMEM containing $10 \%$ FCS at $70-80 \%$ confluency were transfected with $5 \mu \mathrm{g}$ of expression vector by using the Superfect reagent (Qiagen, Valencia, CA, USA). Stable transfectants were selected in the presence of G418 (Sigma) at a concentration of $1 \mathrm{mg} \mathrm{ml}^{-1}$. The expression of the antibody construct was determined in the culture medium by quantitative ELISA. For recombinant protein production, transfected 293T cells were expanded to near confluence in selective DMEM medium containing $0.5 \mathrm{mg} \mathrm{ml}^{-1} \mathrm{G} 418,4 \mathrm{mg} \mathrm{ml}^{-1}$ glucose, $10 \%$ fetal bovine serum, $50 \mathrm{U} \mathrm{ml}^{-1}$ penicillin, $50 \mu \mathrm{g} \mathrm{ml}^{-1}$ streptomycin and were then grown for 3-4 days in serum-free medium.

The recombinant fusion protein, henceforth termed Erb-hcAbRNase, secreted by transfected 293T cells, was purified from culture medium by affinity chromatography on a protein A-Ceramic Hyper DF column (Pall Corporation, Port Washington, NY, USA) loaded with $300-500 \mathrm{ml}$ of conditioned medium, washed with 10 volumes of $100 \mathrm{~mm}$ Tris $-\mathrm{HCl}, \mathrm{pH} 8.0$ containing $0.5 \mathrm{M}$ $\mathrm{NaCl}$ and 10 volumes of $10 \mathrm{~mm}$ Tris $-\mathrm{HCl}, \mathrm{pH} 8.0$. The protein eluate was obtained with $50 \mathrm{~mm}$ glycine $\mathrm{pH} 3.0$, and immediately neutralised with $1 / 10$ volume of $1 \mathrm{M}$ Tris $-\mathrm{HCl}$, $\mathrm{pH} 8.0$.

\section{RNase activity and inhibition}

RNase activity was tested with the acid-insoluble RNA precipitation assay as described previously (Bartholeyns et al, 1977) on yeast RNA $\left(8 \mathrm{mg} \mathrm{ml}^{-1}\right)$. RNase zymograms, carried out on SDS-PAGE electropherograms, were performed as previously described (Blank et al, 1982; De Lorenzo et al, 2004a). For inhibition assays, appropriate amounts of Erb-hcAb-RNase and Erb-hRNase were pre-incubated with increasing concentrations of $\mathrm{cRI}$ at $37^{\circ} \mathrm{C}$ for $10 \mathrm{~min}$ before starting the activity test mentioned above. Ribonuclease inhibitor was purchased from Promega (Madison, WI, USA); its concentration was determined as previously described (Naddeo et al, 2005).

\section{ELISA assays}

ErbB2-positive SKBR3 cells and ErbB2-negative A431 control cells, harvested in non-enzymatic dissociation solution (Sigma), were washed and transferred to U-bottom microtitre plates $\left(1 \times 10^{5}\right.$ cells per well). After blocking with PBS containing $6 \%$ bovine serum albumin (BSA), cells were incubated with conditioned medium or purified immunoagents in ELISA buffer (PBS/BSA 3\%) for $90 \mathrm{~min}$. The pelleted cells were washed, resuspended in $100 \mu \mathrm{l}$ of ELISA buffer and incubated with an anti-human IgG (Fc specific) MAb (Sigma) for detection of Erb-hcAb and Erb-hcAb-RNase. The latter was detected also with an anti-HP-RNase IgG antibody followed by HRP-conjugated goat anti-rabbit immunoglobulin. After $1 \mathrm{~h}$, the plates were centrifuged, washed with ELISA buffer and reacted with $3,3^{\prime}, 5,5^{\prime}$-tetramethylbenzidine (Sigma). Binding values were determined from the absorbance at $450 \mathrm{~nm}$, and reported as the mean of at least three determinations (s.d. $\leqslant 5 \%$ ).

\section{Analyses on cell cultures and lysates}

Cells were seeded in 96-well, flat-bottom plates; SKBR3 cells at a density of $1.5 \times 10^{4}$ well; A431 at a density of $5 \times 10^{3}$ well. After incubation at $37^{\circ} \mathrm{C}$ for $72 \mathrm{~h}$ with the protein under test, viable cells were counted by the Trypan blue-exclusion test. Cell viability was determined in triplicate by using methyl tetrazolium test (Sigma) according to the manufacturer's recommendations. The resulting absorbance at $570 \mathrm{~nm}$ was measured in a microplate counter (Multilabel Counter Victor 3, Perkin-Elmer, Waltham, MA, USA). Cell survival was expressed as percentage of viable cells in the presence of the protein under test, with respect to control cultures grown in the absence of the protein. Typically, s.d. were below 5\%.

SKBR3 cell extracts were prepared as previously described (De Lorenzo et al, 2007). Protein concentration was determined by the Bradford colorimetric assay (Sigma), and aliquots of $20 \mu \mathrm{g}$ were run on $12 \%$ SDS - PAGE, followed by electroblotting onto PVDF membranes (Millipore Corporation, Bedford, MA, USA).

Intracellular Erb-hcAb-RNase was detected with anti-HP-RNase or anti-Fc IgGs, respectively, followed by goat anti-rabbit HRP-conjugated IgGs. The signal from secondary antibodies was visualised by enhanced chemiluminescence detection (ECL western blotting detection kit, Amersham Biosciences, Uppsala, Sweden). The signal intensity of reactive bands was measured with a phosphorimager (GS-710, Bio-Rad, Hercules, CA, USA).

\section{ADCC and CDC tests}

Target and control cells were detached from culture dishes with a cell dissociation solution (Sigma) and transferred to round-bottom 96-well plates $\left(2 \times 10^{4}\right.$ cells per well). For ADCC assays, target or control cells were treated with the immunoagents $\left(5 \mu \mathrm{g} \mathrm{ml}^{-1}\right.$ of serum-free medium) and $\mathrm{PBL}$ at $37^{\circ} \mathrm{C}$ for $3-4 \mathrm{~h}$. For CDC assays, cells were incubated at $37^{\circ} \mathrm{C}$ with human serum. Cultures were performed in triplicate in a final volume of $200 \mu \mathrm{l}$. Controls included target cells incubated in the absence of effector cells or in the presence of either serum or immunoagent alone. Tumour cell lysis was determined by measuring the release of lactate dehydrogenase (LDH) using an LDH detection kit (Roche, Mannheim, Germany). Antibody-dependent cellular cytotoxicity or CDC was calculated as the per cent of cytolysis measured in the presence of immunoagent and $\mathrm{PBL}$ or human serum, for ADCC and CDC, respectively, taking as $100 \%$ the maximal $\mathrm{LDH}$ release determined by lysis of target cells with $1 \%$ Triton X-100. Standard deviations were calculated on the basis of the results obtained from three different experiments.

\section{In vivo antitumour activity}

All experiments were performed with 6-week-old female Balb/ cAnNCrlBR mice (Charles River Laboratories, Calco, Italy). The TUBO cells $\left(5 \times 10^{5}\right)$ were suspended in $0.2 \mathrm{ml}$ sterile PBS and injected subcutaneously (day 0 ) in the right paw. At day 7 , when tumours started to appear, the mice were divided into three groups. At day 15, when tumours were clearly detectable, Erb-hcAb-RNase dissolved in PBS was administered i.p. at doses of $1.8 \mathrm{mg} \mathrm{kg}^{-1}$ of body weight for seven times at $72 \mathrm{~h}$ intervals. The second group of animals was treated with equimolar doses $\left(1.3 \mathrm{mg} \mathrm{kg}^{-1}\right.$ of body weight) of Erb-hcAb, dissolved in PBS and administered i.p. for seven times at $72 \mathrm{~h}$ intervals. The third group of control animals was treated with identical volumes of sterile PBS.

To test the effects of Trastuzumab, used as a control, the experiment was repeated on the same model. The TUBO cells $\left(5 \times 10^{5}\right)$ were suspended in $0.2 \mathrm{ml}$ sterile $\mathrm{PBS}$ and injected subcutaneously (day 0 ) in the right paw. When tumours were clearly detectable, Trastuzumab dissolved in PBS was administered i.p. at doses of $2 \mathrm{mg} \mathrm{kg}^{-1}$ for seven times at $72 \mathrm{~h}$ intervals. The second group of control animals was treated with identical volumes of sterile PBS.

During the period of treatment, tumour volumes $(V)$ were measured with caliper and calculated by the formula of rotational ellipsoid $V=A \times B^{2} / 2$ ( $A$ is the axial diameter, $B$ the rotational diameter). All mice were maintained at the animal facility of the Department of Cellular and Molecular Biology and Pathology, University of Naples 'Federico II'. Animal studies were conducted in accordance with the Italian regulation for experimentation on animals. All in vivo experiments were carried out with ethical committee approval and met the standards required by the UKCCCR guidelines (Workman et al, 1998).

\section{Statistical analysis}

Analysis of variance followed by a post hoc Dunnett's $t$-test was used to analyse the data. A $P$-value $<0.05$ was considered to be significant. 


\section{RESULTS}

Production and purification of a novel fully human anti-ErbB2 compact antibody-RNase conjugate

A new human anti-ErbB2 immunoagent was generated by fusing HP-RNase with the fully human anti-ErbB2 compact antibody (Erb-hcAb) (De Lorenzo et al, 2004b). The cDNAs coding for the human compact antibody Erb-hcAb and HP-RNase were amplified by PCR and cloned into the eukaryotic PCMV-ER-myc expression vector. In particular, the cDNA encoding HP-RNase was cloned downstream to the sequence encoding the carboxy terminus of the scFv-Fc (Erb-hcAb) by adding a spacer encoding a 11-amino-acid residue peptide linker (AAASGGPEGGS) to minimise the steric hindrance between the two moieties of the chimeric protein (Figure 1). The recombinant plasmid, sequenced to confirm faithful cloning, was stably transfected in $293 \mathrm{~T}$ (human embryonic kidney) cells, and the recombinant construct was expressed as a secretion product into the culture medium. Once selected by quantitative ELISA assays, the clone producing the highest levels of Erb-hcAb-RNase was used for the production of the chimeric immunoagent, which was then purified by affinity chromatography on a protein A-Ceramic Hyper DF column. The immunoagent was named Erb-hcAb-RNase .

\section{Characterisation of Erb-hcAb-RNase}

When Erb-hcAb-RNase was analysed by SDS-PAGE (Figure 2), it was found to migrate under reducing conditions with the expected molecular size of about $70 \mathrm{kDa}$ (Figure 2, lane B), and as a dimer of about $140 \mathrm{kDa}$ under non-reducing conditions (Figure 2, lane C). This result indicates that the fusion protein is expressed as a disulphide-linked dimer. Western blotting analyses performed with either an anti-human Fc or an anti-HP-RNase antibody demonstrated immunoreactivity of the purified, dimeric protein with a molecular size of $140 \mathrm{kDa}$ (Figure 2, lanes D and $\mathrm{E}$ ).

The fusion protein was then tested for enzymatic activity by a zymogram, developed using yeast RNA as a substrate. As illustrated in Figure 2 lane F, a single active band was detectable, corresponding to the size of Erb-hcAb-RNase. In a parallel assay, the first generation anti-ErbB2 immunoRNase (Erb-hRNase), made up of the Erbicin scFv and HP-RNase, was used as a positive control (data not shown).

The ribonucleolytic activity of the purified Erb-hcAb-RNase was further tested with the acid-insoluble RNA precipitation assay (Bartholeyns et al, 1977), by which the chimeric immunoagent was found to have a specific activity of $730 \pm 25$ units nmol $^{-1}$. This result was confirmed for several preparations of the recombinant fusion protein. Because the specific activity of hErb-hRNase, that is the monomeric anti-ErbB2 immunoRNase, previously tested, is $950 \pm 25$ units nmol $^{-1}$ (De Lorenzo et al, 2004a), we can conclude that Erb-hcAb-RNase retains about $80 \%$ of the activity of the first generation ImmunoRNase.

To determine the sensitivity of Erb-hcAb-RNase to RI inhibition, the enzymatic assays were repeated in the absence or in the presence of increasing concentrations of RI. Erb-hRNase was used as a positive control, as it was previously found to be fully inihibited by RI (De Lorenzo et al, 2007).

As shown in Figure 3, the novel chimeric protein was found to be susceptible of inhibition by RI, even though it was found to be less sensitive than the monomeric immunoRNase. Indeed, the new ImmunoRNase retains a residual $30 \%$ of the enzymatic activity even at a cRI/IR ratio of about 5 , whereas the activity of the monomeric IR is completely inhibited at a cRI/IR ratio of about 1 (Figure 3).

This result could be considered not surprising as the larger molecular size of the antibody fragment of Erb-hcAb-RNase with respect to that of the $s c F v$ in Erb-hRNase could hinder the

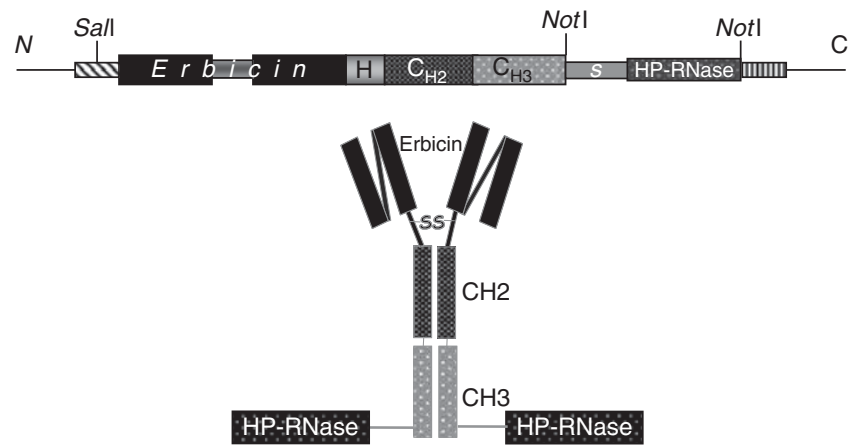

Figure I Schematic representation of the chimeric ImmunoRNase, Erb-hcAb-RNase, obtained by fusing the human compact antibody Erb-hcAb and the HP-RNase. Erbicin=the human anti-ErbB2 scFv; $\mathrm{H}=$ hinge; $\mathrm{CH} 2-\mathrm{CH} 3=$ the heavy constant domains of the human $\mid \mathrm{gGI} F$; $\mathrm{S}=$ the spacer peptide AAASGGPEGGS linking the scFv-Fc and the RNase moieties; HP-RNase $=$ the RNase moiety

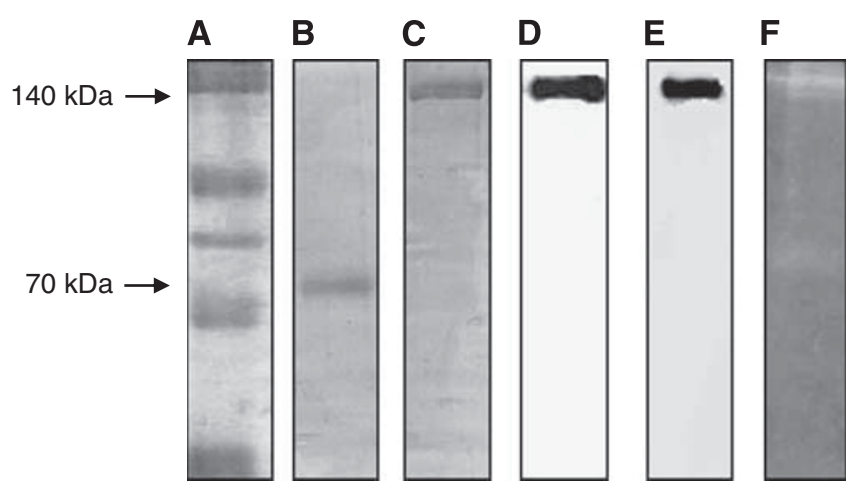

Figure 2 SDS-PAGE and western blotting analyses of purified Erb-hcAb-RNase. Erb-hcAb-RNase was run under reducing (lane B) or non-reducing (lane C) conditions; molecular weight standards are in lane A; western blotting analyses of the purified sample with an anti-human $|g \mathrm{G}|$ (Fc specific) (lane D) or with the anti-HP-RNase antibody (lane E); Zymogram of Erb-hcAb-RNase using yeast RNA as a substrate in lane F.

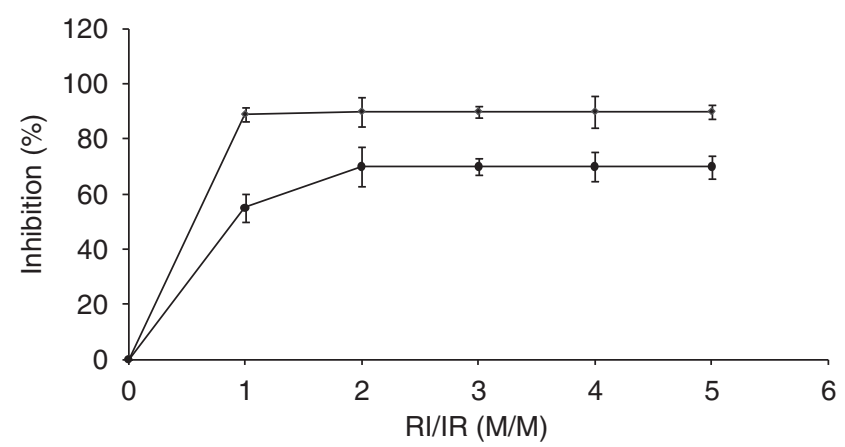

Figure 3 Effects of the RNase inhibitor (RI) on the enzymatic activity of the immunoRNases (IR). Inhibition by RI of the catalytic activity of the antiErbB2 Erb-hcAb-RNase (circles) or Erb-hRNase (rhomboids) was measured at increasing RI/IR ratios.

interactions between RI and the RNase. When the ability of the recombinant fusion protein to bind to ErbB2-positive cells was analysed by ELISA assays (Figure 4), Erb-hcAb-RNase was found to fully retain the specificity and the affinity of the parental compact antibody for mammary carcinoma ErbB2-overexpressing 


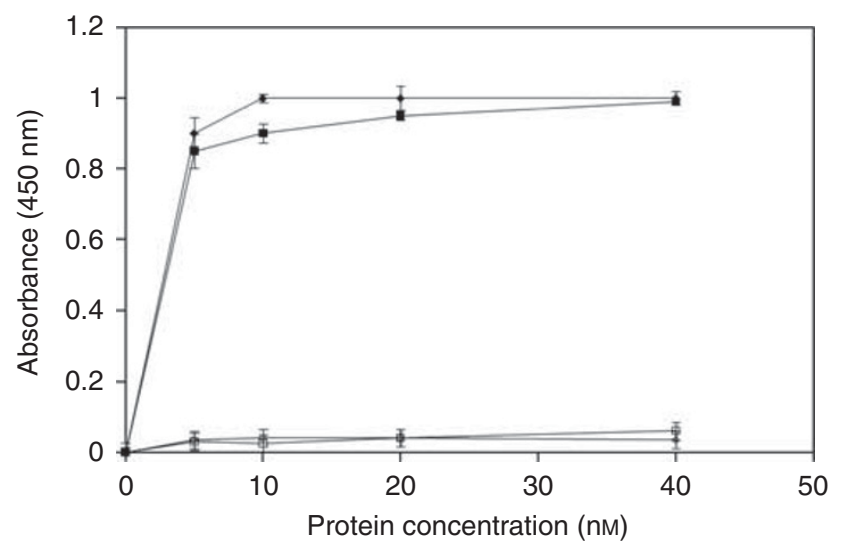

Figure 4 Binding curves of Erb-hcAb-RNase or Erb-hcAb to ErbB2positive (SKBR3) and -negative (A43I) cell lines. SKBR3 cells were tested by enzyme-linked immunoadsorbent assay with Erb-hcAb-RNase (black squares), or with Erb-hcAb (black rhomboids), used as a control; A43I cells were tested as indicated above with Erb-hcAb-RNase or Erb-hcAb (empty squares and rhomboids, respectively).

SKBR3 cells. As a negative control, we used A431 cells (from human epidermoid carcinoma), which express very low levels of ErbB2. The apparent binding affinity of Erb-hcAb-RNase for the ErbB2 receptor, that is the concentration corresponding to halfmaximal saturation, was about $1 \mathrm{~nm}$, identical to that of the parental Erb-hcAb.

These results demonstrate that the compact antibody and the RNase retain their biological functions in the chimeric protein.

\section{Biological effects of Erb-hcAb-RNase on ErbB2-positive tumour cells}

To assess the in vitro effects of Erb-hcAb-RNase on tumour cell growth, the ErbB2-positive SKBR3 and the ErbB2-negative A431 cell lines were incubated with increasing concentrations of Erb-hcAb-RNase, Erb-hcAb or Trastuzumab, used as a control. As shown in Figure 5A, Erb-hcAb-RNase inhibited the growth of SKBR3 cells in a dose-dependent manner, showing an antiproliferative effect more potent than that observed for either the parental Erb-hcAb or Herceptin. The immunoagent did not have any effect on the proliferation of ErbB2-negative A431 cells (see Figure 5A).

These findings suggest that the increased cyotoxicity of Erb-hcAb-RNase with respect to that of Erb-hcAb is due to its RNase moiety, which can exert its enzymatic activity upon internalisation mediated by the antibody moiety.

To test the ability of the immunoRNase to be internalised by ErbB2-positive cells, we analysed the level of Erb-hcAb-RNase in the cytosol of treated cells. Briefly, SKBR3 cells were treated with the immunoRNase $(100 \mathrm{~nm})$ for $16-48 \mathrm{~h}$ at $37^{\circ} \mathrm{C}$, stripped of surfacebound protein with a low $\mathrm{pH}$ glycine $/ \mathrm{NaCl}$ buffer and lysed.

Equal protein amounts of cell extracts were analysed by immunoblotting using either anti-Fc or anti-HP-RNase IgGs, followed by HRP-conjugated secondary antibodies. A strong immunoreactive band with the molecular weight expected for the immunoRNase was observed in the intracellular fraction of treated cells (see Figure 5B), whereas no signal was detected in the extracts of untreated control cells.

These results indicate that the immunoRNase is internalised by ErbB2-positive cells and reaches the cytosol, where potential RNA targets are, so that the RNase can express its cytotoxic action.

To investigate whether Erb-hcAb-RNase was capable of recruiting immune effector functions in vitro, assays for cytolysis of

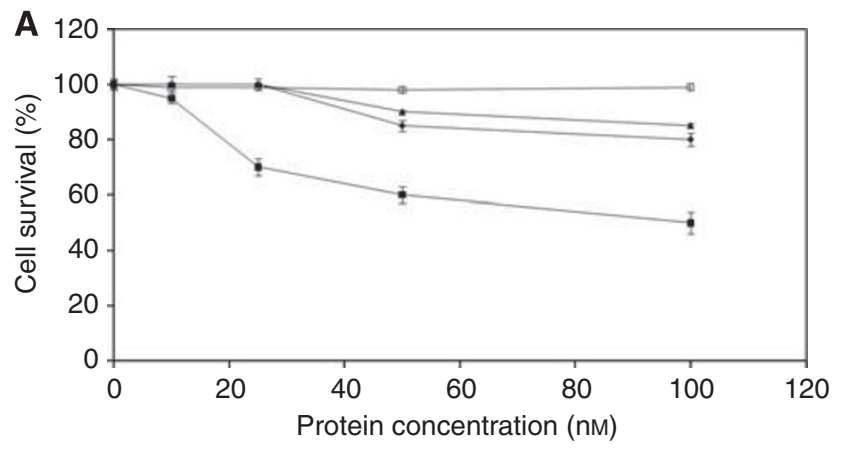

B

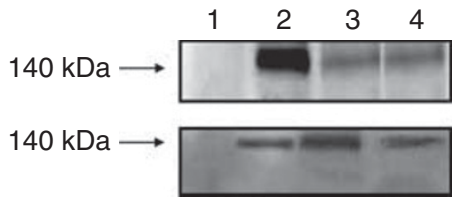

Figure 5 In vitro effects of Erb-hcAb-RNase on tumour cells. (A) Doseresponse curves of ErbB2-positive SKBR3 (black symbols) and ErbB2negative A43I cells (empty symbols), treated for $72 \mathrm{~h}$ with Erb-hcAbRNase (squares). The effects of Erb-hcAb (rhomboids) and Trastuzumab (triangles), used as a positive control, are also shown. (B) Intracellular levels of Erb-hcAb-RNase. The internalisation of Erb-hcAb-RNase by target cells was measured (three experiments) by western blotting with an anti-RNase (upper panel) and an anti-Fc antibody (lower panel). Lanes I-4 (both panels): immunoreactive proteins in the cytosolic fraction of cells untreated (lane I) or treated with Erb-hcAb-RNase (for: $16 \mathrm{~h}$, lane 2; $24 \mathrm{~h}$, lane 3; $48 \mathrm{~h}$, lane 4)

tumour cells as induced by PBL, or complement, were performed. To determine the capacity of Erb-hcAb-RNase to selectively trigger ADCC towards antigen-expressing cells, SKBR3 and A431 cells were incubated for $3 \mathrm{~h}$ with increasing amounts of effector PBL in the absence or in the presence of Erb-hcAb-RNase $\left(5 \mu \mathrm{g} \mathrm{ml}^{-1}\right)$. We used as positive controls both Erb-hcAb and Trastuzumab. As shown in Figure 6A, Erb-hcAb-RNase lysed SKBR3 target cells in the presence of PBL with an efficacy slightly lower than that of the parental Erb-hcAb. The extent of lysis reached almost $80 \%$ of treated cells at a ratio of 100:1 (effector to target cells), whereas Trastuzumab induced about $65 \%$ lysis at the same ratio. No effects were detected when Erb-hcAb was replaced by the parental anti-ErbB2 scFv, lacking the Fc domain or when Erb-hcAb-RNase was tested in parallel assays carried out with ErbB2-negative A431 cells (data not shown), thus demonstrating the specificity of the Erb-hcAb-RNase-dependent cell-mediated cytolytic activity (see Figure 5).

Thus, we can conclude that the presence of the RNase in the new construct does not affect significantly the interactions between the $\mathrm{Fc}$ region of Erb-hcAb and the $\mathrm{CD} 16$ receptor of the natural killer cells.

To test the ability of Erb-hcAb-RNase of inducing CDC against ErbB2-positive tumour cells, SKBR3 target cells were incubated for $6 \mathrm{~h}$ with Erb-hcAb-RNase ( 10 or $30 \mu \mathrm{g} \mathrm{ml}^{-1}$ ) in the absence or in the presence of human serum as a source of complement. As illustrated in Figure 6B, Erb-hcAb-RNase was found to effectively lyse SKBR3 cells in the presence of serum with an average specific lysis of $40 \%$ after $6 \mathrm{~h}$, whereas the parental Erb-hcAb induced about $60 \%$ lysis. Complement-dependent cytotoxicity was not detected when the parental anti-ErbB2 scFv, lacking the Fc domain, was used as a negative control (see Figure 6B), or when ErbB2-negative A431 cells were incubated with Erb-hcAb-RNase and human serum (data not shown). Similarly, as previously reported (Drebrin et al, 1988; De Lorenzo et al, 2004b), no lysis was detectable when SKBR3 cells were treated with Trastuzumab in the presence of human serum (see Figure 6B). 
A

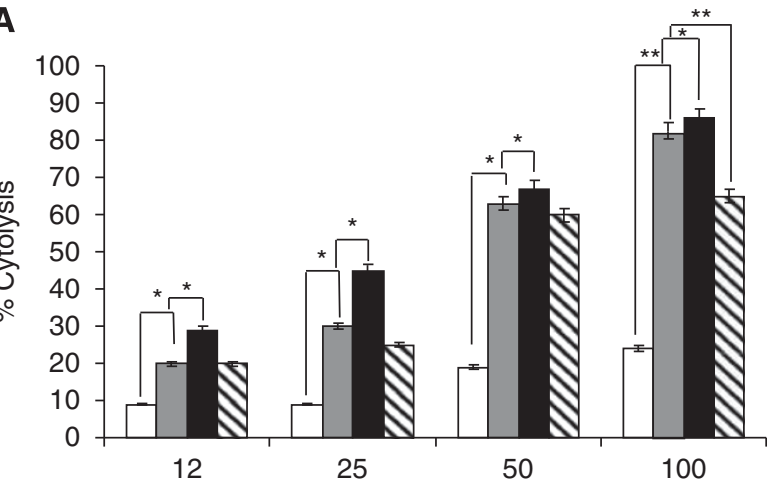

PBL: target cells

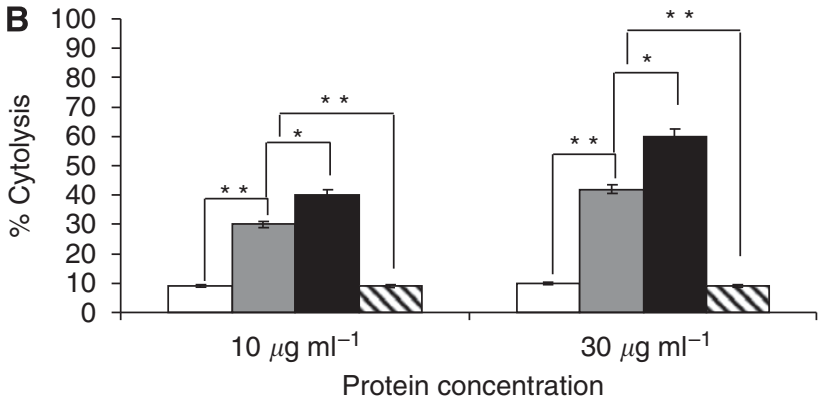

Figure 6 Antibody-dependent and CDC assays of Erb-hcAb-RNase. (A) SKBR3 cells treated with PBL as effector cells at four different ratios in the presence of Erb-hcAb-RNase (grey bars), Erb-hcAb (black bars), Trastuzumab (striped bars) used as positive controls or Erbicin, the parental anti-ErbB2 scFv (white bars), used as a negative control. Histogram represents a summary data of multiple experiments. Bars represent means \pm s.d. $(* P<0.05$; $* * P<0.0$ I). (B) SKBR3 cells were incubated for $6 \mathrm{~h}$ in the presence of human serum as a source of complement with Erb-hcAb-RNase (grey bars), Erb-hcAb (black bars), used as a positive control, Herceptin (striped bars) or Erbicin (white bars), used as a negative control, at concentrations of 10 and $\left.30 \mu \mathrm{g} \mathrm{ml}\right|^{-1}$. Data are reported as the mean of three independent experiments. Bars represent means \pm s.d. $(* P<0.05 ; * * P<0.0$ I)

\section{In vivo antitumour effects of Erb-hcAb-RNase}

For in vivo studies, Erb-hcAb-RNase was tested on murine TUBO tumour cells expressing ErbB2 of rat origin (Rovero et al, 2000). When administered to female mice, TUBO cells induce tumours very similar to the alveolar-type human lobular mammary carcinomas (Di Carlo et al, 1999).

In order to compare the in vivo antitumour efficacy of this novel immunoagent with that of the parental Erb-hcAb, the effects of equimolar doses of Erb-hcAb and Erb-hcAb-RNase were tested in parallel on the same experimental model. In particular, to detect the differential potency of the two immunoagents, they were administered at lower doses than those used for peritumoural administrations of Erb-hcAb in the previous experiment (De Lorenzo et al, 2004b) when a dramatic reduction (96\%) in tumour volume was observed. As shown in Figure 7, the treatment of mice bearing TUBO tumours with seven doses, at $72 \mathrm{~h}$ intervals, of $1.8 \mathrm{mg} \mathrm{kg}^{-1}$ of Erb-hcAb-RNase induced a significant reduction (about 50\%) in tumour volume and showed more potent antitumour effects than those observed for the parental Erb-hcAb (doses of $1.3 \mathrm{mg} \mathrm{kg}^{-1}$ ).

As a further control, we tested in a parallel experiment the effects of equimolar doses of Trastuzumab on the same experimental model. As shown in Figure 7B, Trastuzumab was found to have a very limited inhibitory action on the growing tumours with respect to that of Erb-hcAb-RNase. This may be interpreted by
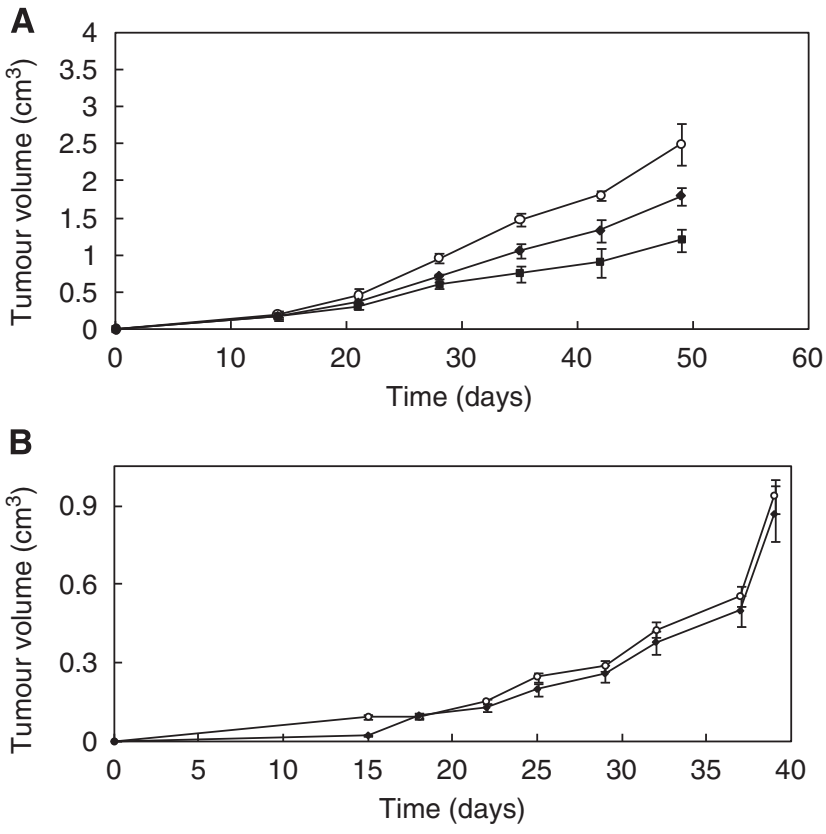

Figure 7 In vivo antitumour effects of Erb-hcAb-RNase and Trastuzumab. (A) In vivo antitumour effects of Erb-hcAb-RNase. Tumour growth was followed in mice inoculated s.c. with $5 \times 10^{5}$ TUBO mammary carcinoma cells. Control animals (empty circles) were treated with sterile PBS solution. Treated animals were injected with Erb-hcAb-RNase (black squares), starting at day 14. Seven doses, each of $1.8 \mathrm{mg} \mathrm{kg}^{-1}$ of body weight, were administered at $72 \mathrm{~h}$ intervals i.p. In a parallel experiment, ErbhcAb (black rhomboids) was administered at equimolar doses $\left(1.3 \mathrm{mg} \mathrm{kg}^{-1}\right)$ as indicated for Erb-hcAb-RNase, for comparison. (B) In vivo antitumour effects of Trastuzumab. Tumour growth was followed in mice inoculated s.c. with $5 \times 10^{5}$ TUBO mammary carcinoma cells. Control animals (empty circles) were treated with sterile PBS solution. Treated animals (black rhomboides) were injected with Trastuzumab, starting at day 14. Seven doses, each of $2 \mathrm{mg} \mathrm{kg}^{-1}$ of body weight, were administered at $72 \mathrm{~h}$ intervals i.p.

considering that humanised Trastuzumab maintains at the antigen-binding site structural determinants of mouse origin, which engender a poor fit with the rat antigen, a negative feature, which is not shared by the human antigen-binding structure of Erb-hcAb.

During the period of treatment, the animals did not show signs of wasting or other visible signs of toxicity.

\section{DISCUSSION}

Immunotoxins, based on toxins fused to antibody moieties specifically reactive to a certain type of tumour cells, have been designed for a novel approach in anticancer therapy. However, the non-specific toxicity of ITs associated to vascular leak syndrome and/or hepatotoxicity, as well as the immunogenicity of their bacterial or plant toxins, have often limited the outcome of ITs as anticancer drugs (Weiner et al, 1989; Reiter and Pastan, 1998; Schindler et al, 2001).

To circumvent these problems, IRs, based on the IT principle, have been proposed (Rybak and Newton, 1999; De Lorenzo et al, 2002a; De Lorenzo and D'Alessio, 2008). In the IRs, the toxin moiety of ITs is replaced by a non-toxic and non-immunogenic RNase, which becomes toxic only upon its internalisation mediated by the antibody moiety in the target cells.

We previously reported on a fully human immunoRNase obtained by fusing a human scFv and a human RNase (De Lorenzo et al, 2004a), named Erb-hRNase, which is directed to the 
ErbB2 receptor, overexpressed in many carcinomas, especially in breast carcinoma (Slamon et al, 1987; Nahta et al, 2006).

Erb-hRNase was found to exert a powerful and selective antitumour action in vitro and in vivo. However, its successful therapeutic use could be somewhat limited by the lack of antibody effector functions, as well as by its monovalent nature and by its small size $(<50 \mathrm{kDa})$, which could be responsible for a reduced tumour retention and a faster clearance from the blood circulation, respectively.

In this study, we report on the production and characterisation of a novel second generation fully human IR targeting the ErbB2 receptor called Erb-hcAb-RNase.

It results from the fusion of the fully human anti-ErbB2 compact antibody Erb-hcAb (De Lorenzo et al, 2004b) with HP-RNase. We have shown here that both the antibody and RNase moieties preserve their biological actions in the immunoconjugate. This second generation immunoRNase is endowed with the following properties:

1. it recognises one of the most specific tumour-associated antigens, such as ErbB2, with an affinity comparable with that of the parental compact antibody;

2. it retains the enzymatic activity of the first generation immunoRNase (Erb-hRNase) but, at difference with the monovalent IR, it is only partially susceptible to RI inhibition;

3 . it displays effective antibody effector functions with an efficacy comparable with that of the parental Erb-hcAb;

4. it inhibits efficiently the proliferation of ErbB2-positive tumour cells both in vitro and in vivo with antitumour effects more potent than those observed for the parental compact antibody. The latter results can be explained by the additional toxic action of the internalised RNase;

5. the size of Erb-hcAb-RNase should be better suited for therapeutic applications due to the potential prolonged halflife with respect to the first generation scFv-based IR and to better penetration properties than full size IgG-toxin immunoconjugates.

It has been already reported by Menzel et al (2008) on a powerful anti-CD30 scFv-Fc-RNase, made up of a CD30 lymphoma specific human scFv-Fc fused to HP-RNase, showing specific binding and in vitro cytotoxicity on CD30 + lymphoma cells at nM concentrations. However, this IR was not tested for its effector functions (ADCC and CDC) and for its in vivo antitumour activity.

To our knowledge, Erb-hcAb-RNase is to date the first fully human antibody-RNase to be constructed and tested with satisfactory results in vivo demonstrating for the first time that the presence of the RNase does not hinder the antibody effector functions (ADCC and CDC).

On the basis of its fully human nature and selectivity of its antitumour action on target cells, Erb-hcAb-RNase represents a promising valuable tool in cancer therapy, thus supporting the hypothesis that the $\mathrm{scFv}-\mathrm{Fc}-\mathrm{RNase}$ format is the most appropriate for the production of a novel generation of IR better suited for therapeutic applications, as it combines the advantages of the first generation IR with those of functional relevant antibody domains. However, the efficacy of this immunoRNase could be further improved for a more efficient killing, as required for a successful cancer therapy, by replacing the native human RNase with a more cytotoxic variant resistant to the RI.

\section{ACKNOWLEDGEMENTS}

We thank Dr Philip Cunnah (Biotecnol SA, Portugal) for kindly providing the anti-ErbB2 compact antibody Erb-hcAb produced by PER.C6 cells, and Dr Salvatore Sequino for his skilled assistance. This work was financially supported by AIRC (Associazione Italiana per la Ricerca sul Cancro), Italy; MUR (Ministero dell’Università e della Ricerca), Italy.

\section{REFERENCES}

Adams GP, Weiner LM (2005) Monoclonal antibody therapy. Nat Biotechnol 23: $1147-1157$

Agus DB, Scher HI, Higgins B, Fox WD, Heller G, Fazzari M, Cordon-Cardo C, Golde DW (1999) Response of prostate cancer to anti-Her-2/neu antibody in androgen-dependent and -independent human xenograft models. Cancer Res 59: 4761-4764

Bartholeyns J, Wang D, Blackburn P, Wilson G, Moore S, Stein WH (1977) Explanation of the observation of pancreatic ribonuclease activity at $\mathrm{pH}$ 4.5. Int J Pept Protein Res 10: $172-175$

Blank A, Sugiyama RH, Dekker CA (1982) Activity staining of nucleolytic enzymes after sodium dodecyl-sulfate-polyacrylamide gel electrophoresis: use of aqueous isopropanol to remove detergent from gels. Anal Biochem 120: $267-275$

Carter P (2001) Improving the efficacy of antibody-based cancer therapies. Nat Rev Cancer 1: 118-129

De Lorenzo C, Arciello A, Cozzolino R, Palmer DB, Laccetti P, Piccoli R, D'Alessio G (2004a) A fully human antitumor immunoRNase selective for ErbB-2-positive carcinomas. Cancer Res 64: 4870-4874

De Lorenzo C, D’Alessio G (2008) From immunotoxins to immunoRNase. Curr Pharm Biotechnol 9: 210-214

De Lorenzo C, D'Alessio G (2009) Human anti-ErbB2 immunoagentsimmunoRNases and compact antibodies. FEBS J 276: $1527-1535$

De Lorenzo C, Di Malta C, Calì G, Troise F, Nitsch L, D’Alessio G (2007) Intracellular route and mechanism of action of ERB-hRNase, a human anti-ErbB2 anticancer immunoagent. FEBS Lett 581: 296-300

De Lorenzo C, Nigro A, Piccoli R, D’Alessio G (2002a) A new RNase-based immunoconjugate selectively cytotoxic for ErbB2-overexpressing cells. FEBS Lett 516: $208-212$

De Lorenzo C, Palmer DB, Piccoli R, Ritter MA, D’Alessio G (2002b) A new human antitumor immunoreagent specific for ErbB2. Clin Cancer Res 8: $1710-1719$
De Lorenzo C, Tedesco A, Terrazzano G, Cozzolino R, Laccetti P, Piccoli R, D’Alessio G (2004b) A human, compact, fully functional anti-ErbB2 antibody as a novel antitumour agent. Br J Cancer 91: 1200-1204

Di Carlo E, Diodoro MG, Boggio K, Modesti A, Modesti M, Nanni P, Forni G, Musiani P (1999) Analysis of mammary carcinoma onset and progression in HER-2/neu oncogene transgenic mice reveals a lobular origin. Lab Invest 79: $1261-1269$

Drebrin JA, Link VC, Greene MI (1988) Monoclonal antibodies specific for the neu oncogene product directly mediate anti-tumor effects in vivo. Oncogene 2: $387-394$

Gelardi T, Damiano V, Rosa R, Bianco R, Cozzolino R, Tortora G, Laccetti P, D’Alessio G, De Lorenzo C (2010) Two novel human anti-ErbB2 immunoagents are active on trastuzumab-resistant tumours. Br J Cancer 102: 513 - 519

Gong SJ, Jin CJ, Rha SY, Chung HC (2004) Growth inhibitory effects of trastuzumab and chemotherapeutic drugs in gastric cancer cell lines. Cancer Lett 214: 215-224

Harris M (2004) Monoclonal antibodies as therapeutic agents for cancer. Lancet Oncol 5: $292-302$

Jørgensen JT (2010) Targeted HER2 treatment in advanced gastric cancer. Oncology 78: 26-33

Li J, Zhu Z (2010) Research and development of next generation of antibody-based therapeutics. Acta Pharmacol Sin 31: 1198-1207

Menzel C, Schirrmann T, Konthur Z, Jostock T, Dübel S (2008) Human antibody RNase fusion protein targeting CD30+ lymphomas. Blood 111: $3830-3837$

Naddeo M, Vitagliano L, Russo A (2005) Interactions of the cytotoxic RNase A dimers with the cytosolic ribonuclease inhibitor. FEBS Lett 579: $2663-2668$

Nahta R, Yu D, Hung MC, Hortobagyi GN, Esteva FJ (2006) Mechanisms of disease: understanding resistance to HER2-targeted therapy in human breast cancer. Nat Clin Pract Oncol 3: 269-280 
Pastan I, FitzGerald D (1991) Recombinant toxins for cancer treatment. Science 254: $1173-1177$

Reiter Y, Pastan I (1998) Recombinant Fv immunotoxins and Fv fragments as novel agents for cancer therapy and diagnosis. Trends Biotechnol 16: $513-520$

Riccio G, Esposito G, Leoncini E, Contu R, Condorelli G, Chiariello M, Laccetti P, Hrelia S, D'Alessio G, De Lorenzo C (2009) Cardiotoxic effects, or lack thereof, of anti-ErbB2 immunoagents. FASEB J 23: $3171-3178$

Rovero S, Amici A, Carlo ED, Bei R, Nanni P, Quaglino E, Porcedda P, Boggio K, Smorlesi A, Lollini PL, Landuzzi L, Colombo MP, Giovarelli M, Musiani P, Forni G (2000) DNA vaccination against rat her-2/Neu p185 more effectively inhibits carcinogenesis than transplantable carcinomas in transgenic BALB/c mice. J Immunol 165: 5133-5142

Rybak SM, Newton DL (1999) Natural and engineered cytotoxic ribonucleases: therapeutic potential. Exp Cell Res 253: 325-335

Schindler J, Sausville E, Messmann R, Uhr JW, Vitetta ES (2001) The toxicity of deglycosylated ricin A chain-containing immunotoxins in patients with non-Hodgkin's lymphoma is exacerbated by prior radiotherapy: a retrospective analysis of patients in five clinical trials. Curr Opin Oncol 13: $168-175$
Seidman A, Hudis C, Pierri MK, Shak S, Paton V, Ashby M, Murphy M, Stewart SJ, Keefe D (2002) Cardiac dysfunction in the trastuzumab clinical trials experience. J Clin Oncol 20: 1215-1221

Slamon DJ, Clark GM, Wong SG, Levin WJ, Ullrich A, McGuire WL (1987) Human breast cancer: correlation of relapse and survival with amplification of the HER-2/neu oncogene. Science 235: 177-182

Stebbing J, Copson E, O'Reilly S (2000) Herceptin (trastuzumab) in advanced breast cancer. Cancer Treat Rev 26: 287-290

Venkiteshwaran A (2009) Tocilizumab. MAbs 1: $432-438$

Weiner LM, O’Dwyer J, Kitson J, Comis RL, Frankel AE, Bauer RJ, Konrad MS, Groves ES (1989) Phase I evaluation of an anti-breast carcinoma monoclonal antibody 260F9-recombinant ricin A immunoconjugate. Cancer Res 49: $4062-4067$

Workman P, Twentyman P, Balkwill F, Balmain A, Chaplin D, Double J, Embleton J, Newell D, Raymond R, Stables J, Stephens T, Wallace J (1998) United Kingdom Co-ordinating Committee on Cancer Research (UKCCCR) Guidelines for the welfare of animals in experimental neoplasia. Br J Cancer 77: 1 - 10

Zhang Q, Chen G, Liu X, Qian Q (2007) Monoclonal antibodies as therapeutic agents in oncology and antibody gene therapy. Cell Res 17: 89-99 